\title{
PRODUÇÃO DE CANA-DE-AÇÚCAR CORRELACIONADA COM O TEOR DE FÓSFORO EM MATO GROSSO DO SUL
}

\section{RAFAEL MONTANARI ${ }^{1}$, PAMELA KERLYANE TOMAZ ${ }^{1}$, DOUGLAS MARTINS PEREIRA PELLIN ${ }^{2}$}

\footnotetext{
1 UNESP/Ilha Solteira, SP, montanari@agr.feis.unesp.br, pamelazootecnia@hotmail.com. ${ }^{2}$ UFGD/Dourados, MS, douglas.pellin@agronomo.eng.br
}

RESUMO: A caracterização da variabilidade espacial de atributos envolvidos nos sistemas de produção é fundamental para o planejamento estratégico do setor sucroenergético brasileiro. Assim, o objetivo foi avaliar a correlação espacial e linear entre os atributos da composição tecnológica da cana-de-açúcar correlacionados com o teor de fosfóro de um Latossolo Vermelho distroférrico, no município de Rio Brilhante, MS. Foi instalada uma malha geoestatística, para a coleta de dados do solo e planta, com 80 pontos amostrais, numa área de 80 ha. A análise de regressão linear indicou que a variabilidade da produtividade da cana-de-açúcar $\left(\mathrm{t} \mathrm{ha}^{-1}\right)$ pode ser explicada pelo teor de fósforo disponível do solo. A análise dos semivarigromas cruzados para os componentes de produção indicou que variável atributo de planta açúcares totais recuperáveis pôde ser estimada por meio da técnica de co-krigagem em função do teor de fósforo do solo. Assim, valores superiores a $23 \mathrm{mg} \mathrm{dm}^{-3}$ desse atributo do solo indicaram sítios com as mais elevadas produtividades da cultura da cana-de-açúcar.

PALAVRAS-CHAVE: Saccharum oficinarum spp, variabilidade espacial, fertilidade do solo.

\section{SUGARCANE YIELD CORRELATED WITH PHOSPHORUS CONTENT IN MATO GROSSO DO SUL}

\begin{abstract}
The spatial variability characterization of involved attributes in production systems is critical to the Brazilian sugarcane industry's strategic planning. Therefore, the aim of this study was to evaluate the linear and spatial correlations among the sugarcane's technological attributes composition correlated with an Oxisol phosphorus content, in Rio Brilhante, Mato Grosso do Sul state. It was installed a geostatistical grid, for soil and plant data collection, with 80 sampling points, in 80 ha. The linear regression analysis indicated that the sugarcane yield $\left(\mathrm{t} \mathrm{ha}^{-1}\right)$ variability can be explained by the phosphorus available content in the soil. The yield components' cross-semivariogram analysis indicated that the plant variable total recoverable sugars could be estimated through co-krigaging technic by the phosphorus content in the soil. Thus, greater values than $23 \mathrm{mg} \mathrm{dm}^{-3}$ of this soil attribute indicated the highest sugarcane productivity sites.
\end{abstract}

KEY WORDS: Saccharum oficinarum spp, spatial variability, soil fertility.

\section{INTRODUÇÃO}

Introduzida no período colonial, a cana-de-açúcar se transformou em uma das principais culturas da economia brasileira. O Brasil não é apenas o maior produtor de cana-deaçúcar, mas também o primeiro do mundo na produção de açúcar e etanol e tem conquistado o 
mercado externo devido à utilização de biocombustíveis como alternativa energética por meio de material renovável. Responsável por mais da metade do açúcar comercializado no mundo, o País deve alcançar taxa média de aumento da produção de 3,25\%, até 2018/19, e colher 47,34 milhões de toneladas do produto, o que corresponde a um acréscimo de 14,6 milhões de toneladas em relação ao período 2007/2008. Para as exportações, o volume previsto para 2019 é de 32,6 milhões de toneladas. O etanol, produzido no Brasil, a partir da cana-de-açúcar, também conta com projeções positivas para os próximos anos, devidas, principalmente, ao crescimento do consumo interno. Assim, a produção projetada para 2019 é de 58,8 bilhões de litros, mais que o dobro da registrada em 2008, consumo interno de 50 bilhões de litros e exportações em 8,8 bilhões (DALCHIAVON et al., 2013).

No estado de Mato Grosso do Sul vem ocorrendo diversas modificações e problemas principalmente com a degradação física por meio do uso intensivo dos solos, acarretando: erosão, perda da fertilidade, redução do conteúdo de matéria orgânica e principalmente, formação de camadas compactadas (ROQUE et al., 2008). O cultivo intensivo do solo sob cana-de-açúcar ao longo dos anos favorece o processo de erosão, pois, com a diminuição da cobertura vegetal, o teor de matéria orgânica diminui, atributo responsável pela melhoria da estrutura do solo (VIEIRA et al., 2011).

Em relação aos três macronutrientes primários, o fósforo é aquele exigido em menores quantidades pelas plantas. Não obstante, trata-se do nutriente mais utilizado na adubação das culturas agrícolas brasileiras, uma vez que sua carência é generalizada nos solos tropicais, assim como pela sua fixação no solo. A maioria dos atributos do solo apresenta dependência espacial, isso significa que os valores assumidos por determinado atributo numa posição definida na área de estudo, variam de acordo com a direção e a distância de separação entre as amostras vizinhas. $\mathrm{O}$ estudo da variabilidade espacial do solo é importante para o planejamento e análise dos setores de pesquisas e produção agropecuárias, pois permite a confecção de mapas de krigagem auxiliando na obtenção de zonas específicas de manejo do solo. Nesse contexto, o uso de técnicas geoestatísticas permite a elaboração de semivariogramas, que quantificam a dependência espacial de atributos do solo e da planta, possibilitando a obtenção de estimativas dessas propriedades em locais não amostrados, com erro mínimo da variância (DALCHIAVON et al., 2013; KAMIMURA et al., 2013). A utilização da geoestatística tem auxiliado na caracterização da variabilidade espacial de atributos do solo, que é aplicada à Ciência do Solo (BURGESS; WEBSTER, 1980), o que auxilia na escolha, locação do talhão e interpretação dos resultados (VIEIRA et al., 2011).

A geoestatística baseia-se na teoria das variáveis regionalizadas, que leva em consideração as componentes aleatórias e estruturais da dependência espacial das variáveis, fornecendo ferramentas quantitativas para a sua descrição e estimativas sem tendência. Devido à importância prática da agricultura de precisão, ultimamente no Brasil os estudos geoestatísticos dos atributos químicos do solo relacionados com os componentes de produção das culturas vem se intensificando (SOUZA et al., 2010a,b; VIEIRA et al., 2011; DALCHIAVON et al., 2012; MONTANARI et al., 2012; CAMPOS et al., 2013). Assim, o objetivo foi avaliar a correlação espacial e linear entre os atributos da composição tecnológica da cana-de-açúcar correlacionados com o teor de fósforo de um Latossolo Vermelho distroférrico, no município de Rio Brilhante, MS.

\section{MATERIAL E MÉTODOS}

O estudo foi realizado durante os ciclos de cana planta (2003/2004) e cana soca (2004/2011). A área experimental, de propriedade da Usina ETH Bioenergia S/A se localiza nas coordenadas $21^{\circ} 40^{\prime} 10^{\prime \prime}$ de latitude Sul e 53 $50^{\prime} 15^{\prime \prime}$ de longitude oeste com elevação de 
312 m acima do nível do mar, no município de Rio Brilhante, estado de Mato Grosso do Sul MS. O trabalho foi desenvolvido num talhão de cana-de-açúcar (80 ha), no sistema de colheita mecanizada sem queima prévia (cana crua), em solo classificado como Latossolo Vermelho distróférrico com textura argilosa (EMBRAPA, 2006). O clima da região foi classificado como Cwa, mesotérmico úmido, verões quentes e invernos secos, com precipitação anual média de $1.200 \mathrm{~mm}$, com temperatura do mês mais frio inferior a $18{ }^{\circ} \mathrm{C}$ e a do mês mais quente superior a $22{ }^{\circ} \mathrm{C}$ (FIETZ et al., 2008).

O plantio do talhão foi realizado no dia 04 de março de 2003 , utilizando-se dois colmos de cana (colmo-semente) do cultivar RB-85-5536, depositados no sulco de plantio e posteriormente cortados, de modo que os toletes (rebolos) com três a quatro gemas permanecessem paralelos (pé com ponta), proporcionando a distribuição de 15 a 18 gemas viáveis por metro de sulco. A adubação de plantio consistiu na adição de $25 \mathrm{~kg} \mathrm{ha}^{-1}$ de $\mathrm{N}$ na forma de ureia, $150 \mathrm{~kg} \mathrm{ha}^{-1}$ de $\mathrm{K}_{2} \mathrm{O}$ na forma de cloreto de potássio $(\mathrm{KCl})$ e $75 \mathrm{~kg} \mathrm{ha}^{-1}$ de $\mathrm{P}_{2} \mathrm{O}_{5}$ na forma de superfosfato simples, calculados com base nos resultados da análise de solo. Após 10 dias do corte da cana planta realizou-se a aplicação mecanizada de $140 \mathrm{~kg} \mathrm{ha}^{-1} \mathrm{de} \mathrm{N}$ na forma de ureia e $110 \mathrm{~kg} \mathrm{ha}^{-1}$ de $\mathrm{K}_{2} \mathrm{O}$ na forma de cloreto de potássio $(\mathrm{KCl})$ ao lado da linha de cana.

Um croqui georreferenciado foi confeccionado contendo 80 pontos distribuídos aleatoriamente de forma a cobrir a área de 80 ha do talhão escolhido para a condução do experimento. As coordenadas geraram uma planilha de dados de latitude e longitude de cada ponto no espaço. Com o auxílio de um GPS de bolso, Garmin etrex, alimentado por essa planilha de coordenadas, os pontos foram materializados num talhão de cana-de-açúcar (Figura 1).

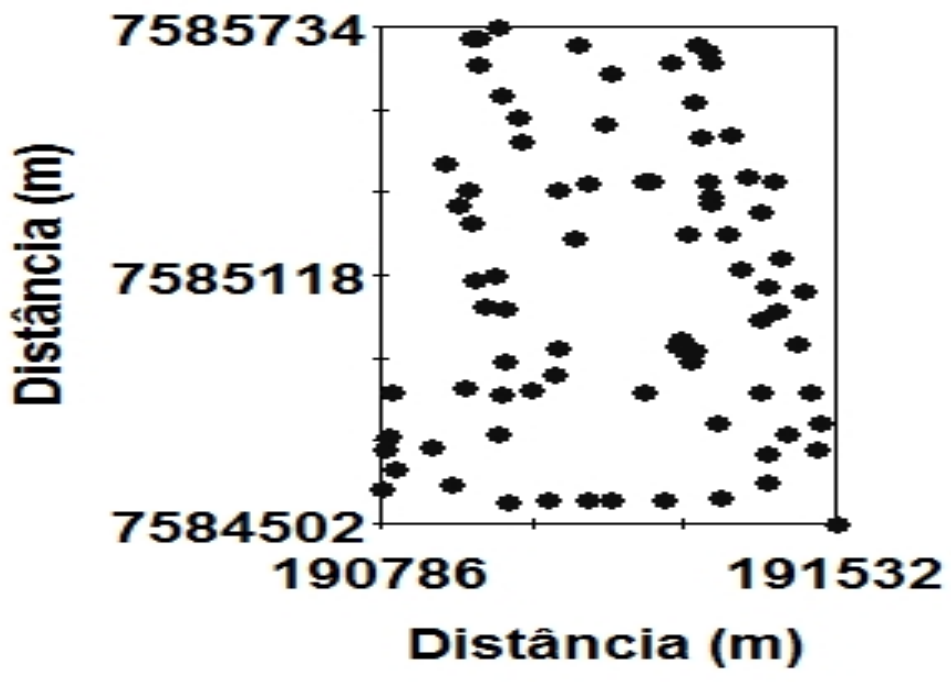

Figura 1. Representação esquemática do grid de amostragem.

Para a coleta dos atributos da planta foi definido uma área útil de duas linhas de $3 \mathrm{~m}$ de cultivo, totalizando $9 \mathrm{~m}^{2}$ ao redor do ponto amostral. Para a medida do atributo tonelada de cana-de-açúcar por hectare ( $\mathrm{TCH})$, utilizou-se uma balança eletrônica portátil com capacidade de $50 \mathrm{~kg}$ e precisão de $20 \mathrm{~g}$, para tanto, os colmos foram cortados manualmente, posteriormente organizados em feixes, e mensurado sua massa. Para a obtenção dos dados tecnológicos da planta, após medida do $\mathrm{TCH}$, foram retirados 10 colmos industrializáveis, retirados em sequência na touceira, os feixes foram despontados, despalhados, etiquetados e 
encaminhados ao laboratório da Usina Eldorado ETH - Bioenergia S/A, onde foram determinados os açúcares totais recuperáveis (ATR) seguindo a metodologia Consecana (2006). O teor de fósforo disponível no solo (P) foi determinado conforme Raij et al. (1991) para as profundidades de 0,00-0,20 m (P1) e 0,20-0,40 m (P2). Optou-se estudar até a profundidade de $0,40 \mathrm{~m}$, pois é a camada mais susceptível às alterações físicas do solo, no qual o sistema radicular atua com maior intensidade.

Para cada atributo estudado, efetuou-se a análise descritiva clássica, com auxílio do software estatístico SAS (versão 9, SAS Institute, Cary, NC, EUA), em que foram calculados a média, mediana, valores mínimos e máximos, desvio padrão, coeficiente de variação, curtose, assimetria e distribuição de frequência. Posteriormente, realizou-se a identificação dos outliers, efetuando a substituição dos seus valores pelo valor médio dos circunvizinhos contidos na malha. Para testar a hipótese de normalidade ou de log normalidade, foi utilizada a estatística de Shapiro e Wilk ao nível de 0,05 de probabilidade. Em adição, foi realizada a análise de correlação linear simples entre todos os atributos estudados, anteriormente às análises de regressões lineares para os pares de maior interesse.

A dependência espacial dos atributos estudados foi determinada por meio do cálculo do semivariograma experimental. Contudo, para aqueles atributos que apresentaram interdependência espacial, foram calculados os semivariogramas cruzados, com base nos pressupostos de estacionaridade da hipótese intrínseca, utilizando-se o programa $\mathrm{GS}^{+}$ (Gamma Design Software, LLC, Plainwell, MI, EUA).

Os ajustes dos modelos matemáticos aos semivariogramas experimentais e cruzados foram efetuados prioritariamente pela seleção inicial de: (a) menor soma dos quadrados dos desvios (SQR); (b) maior coeficiente de determinação $\left(\mathrm{r}^{2}\right)$; e (c) maior avaliador da dependência espacial (ADE). A decisão final do modelo que representou o ajuste foi atestada pela validação cruzada, assim como para a definição do tamanho da vizinhança que proporcionou a melhor malha de krigagem. Para cada atributo foram relacionados o efeito pepita $\left(\mathrm{C}_{0}\right)$, o patamar $\left(\mathrm{C}_{0}+\mathrm{C}\right)$ e o alcance $\left(\mathrm{A}_{0}\right)$. A análise do avaliador da dependência espacial (ADE) foi efetuada conforme a Eq. 1:

$\mathrm{ADE}=\frac{\mathrm{C}}{\mathrm{C}+\mathrm{C}_{0}} \times 100$

sendo:

ADE - avaliador da dependência espacial

C - a variância estrutural

$\mathrm{C}+\mathrm{C}_{0}$ - o patamar

A interpretação proposta para o ADE foi a seguinte: a) ADE < 20\% = variável espacial de muito baixa dependência (MB); b) $20 \% \leq \mathrm{ADE}<40 \%$ = baixa dependência (BA); c) $40 \% \leq \mathrm{ADE}<60 \%=$ média dependência (ME); d) $60 \% \leq \mathrm{ADE}<80 \%$ = alta dependência (AL) e e) $80 \% \leq \mathrm{ADE}<100 \%$ = muito alta dependência (MA) (DALCHIAVON et al., 2012).

\section{RESULTADOS E DISCUSSÃO}

De acordo com Pimentel-Gomes e Garcia (2002), a variabilidade de um atributo pode ser classificada segundo a magnitude de seu coeficiente de variação $(\mathrm{CV})$, sendo baixa $(\mathrm{CV} \leq$ $10 \%)$, média $(10 \%<\mathrm{CV} \leq 20 \%)$, alta $(20 \%<\mathrm{CV} \leq 30 \%)$ e muito alta $(\mathrm{CV}>30 \%)$, o que implica em precisões experimentais, respectivamente, alta, média, baixa e muito baixa. Portanto, os atributos TCH e ATR apresentaram média variabilidade com valores de 
coeficientes de variação de 19,9 e 14,4\%, respectivamente (Tabela 1). Resultados semelhantes foram obtidos por Souza et al. (2010a) e Souza et al. (2010b) onde os autores trabalharam num Latossolo Vermelho, respectivamente, encontrando valores de 11 a 18,8\%.

Tabela 1. Estatística descritiva dos componentes tecnológicos da cultura da cana-de-açúcar e do teor de fósforo de um Latossolo Vermelho distrófico.

\begin{tabular}{lcccccccccc}
\hline Atributo & ME & MD & MA & MI & DP & CV $(\%)$ & CT & AM & Pr<w & DF \\
\hline TCH $\left(\mathrm{t} \mathrm{ha}^{-1}\right)$ & 59,64 & 59,11 & 101,11 & 31,9 & 11,910 & 19,9 & 1,114 & 0,695 & 0,065 & NO \\
ATR $\left(\mathrm{kg} \mathrm{t}^{-1}\right)$ & 105,9 & 106,8 & 137,3 & 55,13 & 15,230 & 14,4 & 0,523 & $-0,351$ & 0,087 & NO \\
P1 $\left(\mathrm{mg} \mathrm{dm}^{-3}\right)$ & 3,16 & 3,00 & 7,00 & 1,00 & 1,479 & 46,7 & - & - & - & ND \\
P2 $\left(\mathrm{mg} \mathrm{dm}^{-3}\right)$ & 2,79 & 2,0 & 23,0 & 1,0 & 1,569 & 48,4 & - & - & - & ND \\
\hline
\end{tabular}

TCH - Tonelada de colmo por hectare; ATR - Açúcares totais recuperáveis; P - Teor de fósforo, coletados nas camadas do solo de 0,00-0,20 m e 0,20-0,40 m; DF - Distribuição de frequência; NO - Normal; ND - Não determinada; ME= média; MD = Mediana; $\mathrm{MA}=$ Máximo; $\mathrm{MI}=$ Mínimo; $\mathrm{DP}=$ Desvio Padrão; $\mathrm{CV}=$ coeficiente de variação; $\mathrm{CT}=$ curtose; $\mathrm{AM}=$ assimetria.

Para os teores de fósforo do solo nas diferentes profundidades P1 e P2, apresentaram variabilidade espacial muito alta com coeficientes de variação de 46,7 e 48,4\%, respectivamente. Dalchiavon et al. (2012) estudando atributos químicos de um latossolo de Cerrado também encontraram muito alta a variabilidade para P1 e P2, sendo de 57,82 e $87,61 \%$, respectivamente. Quando uma variável possuir distribuição de frequência do tipo normal, a medida de tendência central mais adequada para representá-la deve ser a média. Em contrapartida, caso possua distribuição de frequências do tipo lognormal a medida de tendência central mais adequada será a mediana (DALCHIAVON et al., 2012). Os atributos de planta TCH e ATR apresentaram distribuição de frequência do tipo normal, com valores do teste de normalidade de Shapiro e Wilk de 0,065 e 0,087, respectivamente. Esse tipo de distribuição para atributos de origem biológica é comum (DALCHIAVON et al., 2014), assim esses atributos podem ser representados por seus valores médios. Esses dados corroboram com os de Dalchiavon et al. (2012) para o teor de fósforo do solo.

Ainda na Tabela 1 pode-se observar que a média do atributo TCH foi de 59,64 tha-1 valor abaixo da média nacional $\left(70,3 \mathrm{t} \mathrm{ha}^{-1}\right)$ e do estado do Mato Grosso do Sul $\left(70,7 \mathrm{t} \mathrm{ha}^{-1}\right)$ (CONAB, 2012). Esse dado divergiu dos obtidos por Carvalho et al. (2011) avaliando a produtividade de cana-de-açúcar num Latossolo Vermelho distrófico, para análise de custos de produção dos diferentes sistemas de preparo do solo e estudo das possíveis alterações nos atributos físicos e químicos encontraram um alto valor de TCH em cana planta de $(146,20 \mathrm{t}$ $\mathrm{ha}^{-1}$ ), valor esse duas vezes superior ao da média nacional.

Para o atributo tecnológico, ATR observa-se um valor médio de $105,9 \mathrm{~kg} \mathrm{t}^{-1}$ valor considerado abaixo da referência $\left(121,97 \mathrm{~kg} \mathrm{t}^{-1}\right)$ estabelecida por Dalchiavon et al. (2013). O valor médio de ATR do presente estudo também foi abaixo do valor médio de referência do obtido por Rapassi et al. (2009) na região oeste paulista no ano de $2007 \mathrm{de} 144,56 \mathrm{~kg} \mathrm{t}^{-1}$, e $10 \%$ abaixo do valor obtido por Carvalho et al. (2011) onde a coleta da cana-de-açúcar encontrava-se no primeiro corte. A comparação da ATR obtida no presente estudo com a obtida por Carvalho et al. (2011), estando ambas abaixo do valor de referencia estabelecido, nos permite inferir que o número de cortes do presente não influenciou os baixos valores obtidos para este atributo. De acordo com a classe de fertilidade do solo para o teor de fósforo proposta pro Raij (1991), foi possível conhecer a dinâmica do fósforo no presente trabalho. Assim, o P (P1 = 3,16 mg dm$\left.{ }^{-3} ; \mathrm{P} 2=2,79 \mathrm{mg} \mathrm{dm}^{-3}\right)$ apresentou teor muito baixo no solo (Tabela 1). 
A análise de correlação linear simples entre componentes tecnológicos da cultura da cana-de-açúcar e o teor de fósforo no solo nas duas profundidades estudadas indicou que foi significativa $(\mathrm{p}<0,05)$ para os pares: $\mathrm{TCH} \times \mathrm{P} 1(\mathrm{r}=0,250)$; $\mathrm{TCH} \times \mathrm{P} 2(\mathrm{r}=0,247)$ e ATR $\times \mathrm{P} 2$ $(\mathrm{r}=0,247)$, indicando que com um aumento do teor de fósforo no solo haverá um aumento na tonelada de colmos por hectare e nos açúcares totais recuperáveis (Figura 2).

As principais equações de regressão linear simples entre os atributos pesquisados, no âmbito das correlações estabelecidas entre planta e solo estão apresentadas na Figura 2, sendo: a equação de regressão da TCH em função do P1 e P2 foram representadas por um modelo linear com significativos coeficentes de correlação ( $\mathrm{P} 1, \mathrm{r}=0,250^{*}$ e $\mathrm{P} 2, \mathrm{r}=0,247^{*}$ ). Portanto, a variação da tonelada de cana por hectare $(\mathrm{TCH})$ em questão pôde ser explicada por $25 \%$ da variação dos dados do teor de fósforo no solo. Dessa forma, quando o $\mathrm{P}$ variar entre 1 e 7, a TCH aumentará de 55 a $68 \mathrm{t} \mathrm{ha}^{-1}$.
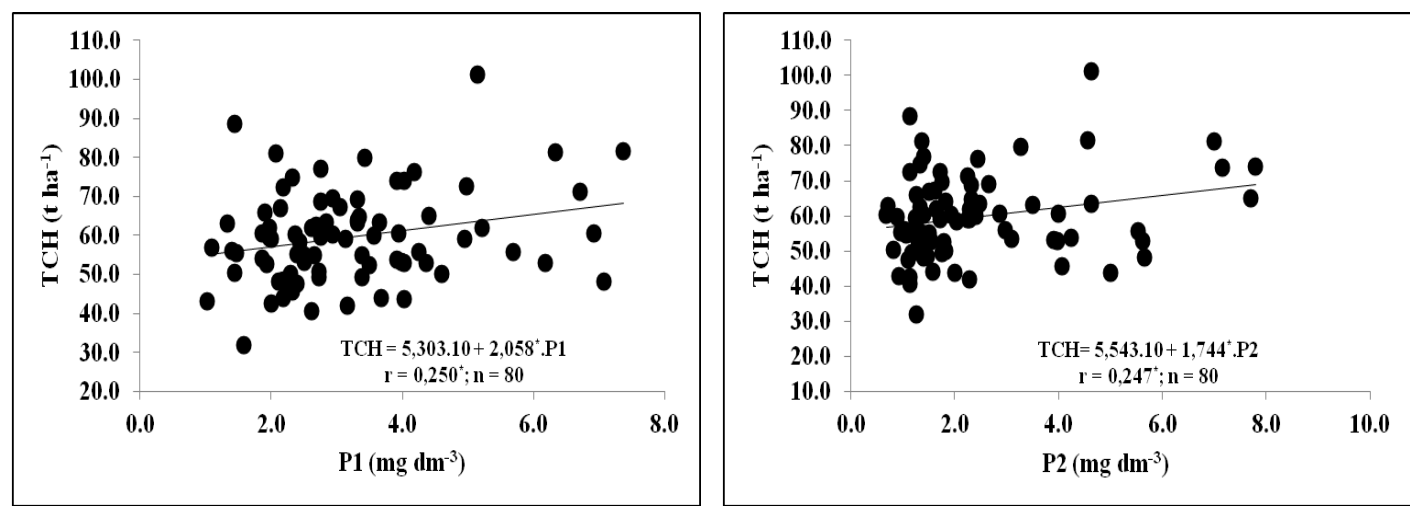

Figura 2. Análise de regressão linear entre a tonelada de cana-de-açúcar por hectare e o teor de fósforo de um Latossolo Vermelho distrófico.

Os atributos TCH, ATR e P2 apresentaram dependência espacial, medida pelo ADE, média para ATR $(\mathrm{ADE}=51,3 \%)$ e $\mathrm{P} 2(\mathrm{ADE}=52,5 \%)$ e muito alta para $\mathrm{TCH}(\mathrm{ADE}=84,3 \%)$, cujos modelos de semivariogramas foram do tipo exponencial, esférico e gaussiano, respectivamente (Tabela 2). Já o P1 apresentou efeito pepita puro. Quanto ao desempenho dos semivariogramas, a relação decrescente deles, analisada pela grandeza do coeficiente de determinação espacial $\left(\mathrm{r}^{2}\right)$, foi a seguinte: 1) P2 $\left.(0,790), 2\right)$ ATR $(0,380)$ e 3$)$ TCH $(0,360)$. Assim, esses dados ficaram basicamente nas mesmas magnitudes de valores que os de Dalchiavon et al. (2012) para o teor de fósforo com um $\mathrm{r}^{2}=0,780$ e $\mathrm{ADE}=50 \%$.

$\mathrm{Na}$ análise de co-krigagem, o enfoque foi para a ATR $=\mathrm{f}(\mathrm{P} 2)$, uma vez que este apresentou semivariograma cruzado com lags distribuídos apenas em um dos quadrantes (primeiro) (Tabela 2 e Figura 3), denotando assim um aspecto confiável entre as variáveis P2 e ATR, apresentando, ainda, um confiável $\mathrm{r}^{2}=0,686$.

A co-krigagem existente entre ATR $\times$ P2 (Tabela 2 e Figura 3) sugeriram P2 como bom indicador da sua qualidade química, quando o objetivo for o de implementar a produtividade da tonelada de cana por hectare. Sendo assim, podem ser recomendadas específicas práticas conservacionistas do solo, dirigidas aos sítios onde a P2 resultou seus menores valores, visando-se elevar a respectiva produtividade de cana-de-açúcar do local. 
Tabela 2. Modelos e parâmetros ajustados aos semivariogramas experimentais e cruzados para os componentes tecnológicos da cana-de-açúcar e o teor de fósforo de um Latossolo Vermelho distrófico.

\begin{tabular}{|c|c|c|c|c|c|c|c|}
\hline Atributos & Modelo & $\mathrm{C}_{0}$ & $\mathrm{C}_{0}+\mathrm{C}$ & $\mathrm{A}_{0}(\mathrm{~m})$ & $\mathrm{r}^{2}$ & SQR & ADE \\
\hline $\mathrm{TCH}\left(\mathrm{t} \mathrm{ha}^{-1}\right)$ & exp. (89) & $1,270.10$ & $8,070.10$ & 246 & 0,36 & $1,937.10$ & 84,3 \\
\hline $\operatorname{ATR}\left(\mathrm{kg} \mathrm{t}^{-1}\right)$ & esf. (51) & $1,027.10$ & $2,109 \cdot 10^{2}$ & 410 & 0,38 & $1,478.10$ & 51,3 \\
\hline $\mathrm{P} 1\left(\mathrm{mg} \mathrm{dm}^{-3}\right)$ & epp & 1,962 & 1,962 & - & - & - & - \\
\hline $\mathrm{P} 2\left(\mathrm{mg} \mathrm{dm}^{-3}\right)$ & gau.(50) & 1,708 & 3,588 & 824 & 0,79 & 2,12 & 52,5 \\
\hline $\mathrm{ATR}=\mathrm{f}(\mathrm{P} 2)$ & gau. (102) & 6,311 & $4,800 \cdot 10^{-1}$ & 686 & 0,686 & $1,830.10$ & 92,4 \\
\hline
\end{tabular}

$\mathrm{TCH}$, ATR e ATR = f(P2) são, respectivamente, a tonelada de colmos por hectare, açúcares totais recuperáveis e açúcar total recuperável em função do fósforo, coletado na profundidade de 0,20-0,40 m; exp - Exponencial; epp - Efeito pepita puro; gau

- Gaussiano; esf - Esférico, modelo sucedido por número entre parêntesis, significando o número de pares no primeiro lag; SQR

- Soma dos quadrados dos resíduos; ADE - Avaliador da dependência espacial
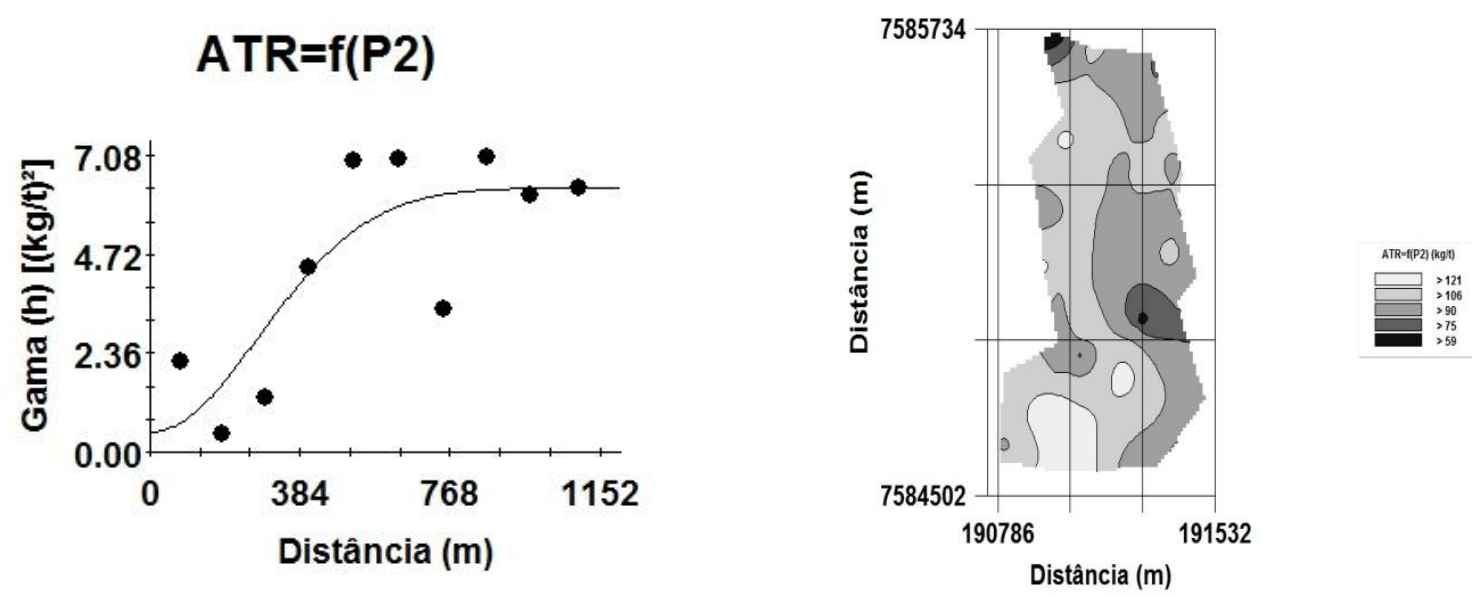

Figura 3. Semivariograma cruzado e mapa de co-krigagem entre o açúcar total recuperável e o teor de fósforo de um Latossolo Vermelho distrófico.

\section{CONCLUSÕES}

Os valores baixos do teor de fósforo no solo, nas faixas de $2,8-3,1 \mathrm{mg} \mathrm{dm}{ }^{-3}$, proporcionaram substancial queda da produtividade da cultura da cana-de-açúcar.

A produtividade da tonelada de cana por hectare foi explicada por um modelo linear direto altamente significativo, dado em função do fósforo do solo.

Os açúcares totais recuperáveis puderam ser estimados por meio da co-krigagem com o teor de fósforo do solo. Assim, valores superiores a $23 \mathrm{mg} \mathrm{dm}^{-3}$ desse atributo do solo indicaram sítios com as mais elevadas produtividades da cultura da cana-de-açúcar.

\section{REFERÊNCIAS BIBLIOGRÁFICAS}

BURGESS, T. M.; WEBSTER, R. Optimal interpolation and isarithmic mapping of soilproperties. I. The semivariogram and punctual kriging. Journal of Soil Science, Amsterdam-HO, v. 31, n. 2, p. 315-331, 1980. 
CAMPOS, M. C. C.; SOARES, M. D. R.; OLIVEIRA, I. A.; SANTOS, L. A. C.; AQUINO, R. E. Spatial variability of physical attributes in Alfisol under agroforestry, Humaitá region, Amazonas state, Brazil. Revista de Ciências Agrárias Amazonian Journal, Belém-PA, v. 56, n. 1, p. 149-159, 2013.

CONAB - COMPANHIA NACIONAL DE ABASTECIMENTO. Cana-de-açúcar. Quadro Comparativo de Área, Produção e Produtividade, Safra 2012/2013 - $1^{\circ}$ Levantamento, Brasília-DF, 2012. 74. Disponível em: <http://www.conab.gov.br/conabweb>. Acesso em: 05 Dez. 2012.

CONSELHO DOS PRODUTORES DE CANA DE AÇÚCAR, AÇÚCAR E ÁLCOOL DO ESTADO DE SÃO PAULO - CONSECANA. Manual de instruções. 5. ed. Piracicaba-SP, 2006. 112 p.

CARVAlHO, L. A.; SILVA JÚNIOR, C. A.; NUNES, W. A. G. A.; MEURER, I.; SOUZA JÚNIOR, W. S. Produtividade e viabilidade econômica da cana-de-açúcar em diferentes sistemas de preparo do solo no Centro-Oeste do Brasil. Revista de Ciências Agrárias, Lisboa-POR, v. 34, n. 1, p. 199-211, 2011.

DALCHIAVON, F. C.; CARVALHO, M. P.; ANDREOTTI, M.; MONTANARI, R. Variabilidade espacial de atributos da fertilidade de um Latossolo vermelho distroférrico sob sistema plantio direto. Revista Ciência Agronômica, Fortaleza-CE v. 43, n. 3, p. 453-461, 2012.

DALCHIAVON, F. C.; CARVALHO, M. P.; MONTANARI, R.; ANDREOTTI, M. Strategy of specification management areas: rice grain yield as related to soil fertility. Revista Brasileira de Ciência do Solo, Viçosa-MG, v. 37, n. 1, p. 45-54, 2013.

DALCHIAVON, F. C.; CARVALHO, M. P.; MONTANARI, R.; ANDREOTTI, M. Interrelações da produtividade de cana soca com a resistência à penetração, umidade e matéria orgânica do solo. Revista Ceres, Viçosa-MG, v. 61, n. 2, p. 255-264, 2014.

EMBRAPA - EMPRESA BRASILEIRA DE PESQUISA AGROPECUÁRIA. Sistema brasileiro de classificação de solos. 2. ed. Rio de Janeiro: EMBRAPA, 2006. 306 p.

FIETZ, C. R.; FISCH, G. F. O. Clima da Região de Dourados, MS. Dourados: Embrapa Agropecuária Oeste, 2008. 32p.

KAMIMURA, K. M.; SANTOS, G. R.; OLIVEIRA, M. S.; DÍAS JUNIOR, M. S.; GUIMARÃES, P. T. G. Variabilidade espacial de atributos físicos de um latossolo vermelho amarelo, sob lavoura cafeeira. Revista Brasileira de Ciência do Solo, Viçosa-MG, v. 37, n. 4, p. 877-888, 2013.

MONTANARI, R.; SOUZA, G. S. A.; PEREIRA, G. T.; MARQUES JÚNIOR, J.; SIQUEIRA, D. S.; SIQUEIRA, G. M. The used of scale semivariograms to plan soil sampling in sugarcane fields. Precision Agriculture, Amsterdam-HO, v. 13, n. 1, p. 542-552, 2012.

PIMENTEL-GOMES, F. P., GARCIA, C. H. Estatística aplicada a experimentos agronômicos e florestais. Piracicaba: FEALQ, 2002. 309 p.

RAIJ, B. VAN. Fertilidade do solo e adubação. Piracicaba-SP: Ceres, 1991. 343 p.

RAPASSI, R. M. A.; TARSINATO, M. A. A.; BOLONHEZI, A. C. Avaliação técnica e econômica de sistemas de produção da cana-de-açúcar (Saccharum spp) na região oeste do estado de São Paulo. Informações Econômicas, v. 39, n. 1, p.11-21, 2009. 
ROQUE, M. W.; MATSURA, E. E.; SOUZA, Z. M.; BIZARI, D. R.; SOUZA, A. L. Correlação linear e espacial entre a resistência do solo ao penetrômetro e a produtividade do feijoeiro irrigado. Revista Brasileira de Ciência do Solo, Viçosa-MG, v. 32, n. 5, p. 1827 1835, 2008.

SOUZA, Z. M.; CERRI, D. G. P.; COLET, M. J.; RODRIGUES, L. H. A.; MAGALHÃES, P. S. G.; MANDONI, R. J. A. Análise dos atributos do solo e da produtividade da cultura de cana-de-açúcar com o uso da geoestatística e árvore de decisão. Ciência Rural, Santa MariaRS, v. 40, n. 4, p. 840-847, 2010a.

SOUZA, Z. M.; MARQUES JÚNIOR, J.; PEREIRA, G. T. Geoestatística e atributos do solo em áreas cultivadas com cana-de-açúcar. Ciência Rural, Santa Maria-RS, v. 40, n. 1, p. 48$56,2010 \mathrm{~b}$.

VIEIRA, S. R.; DECHEN, S. C. F.; SIQUEIRA, G. M.; DUFRANC, G. Variabilidade espacial de atributos físicos e químicos relacionados com o estado de agregação de dois latossolos cultivados no sistema de semeadura direta. Bragantia, Campinas-SP, v. 70, n. 1, p. 185-195, 2011. 